Document downloaded from:

http://hdl.handle.net/10251/56966

This paper must be cited as:

F. Payri; P. Olmeda; Guardiola, C.; J. Martín (2011). Adaptive determination of cut-off frequencies for filtering the in-cylinder pressure in diesel engines combustion analysis. Applied Thermal Engineering. 31:2869-2876. doi:10.1016/j.applthermaleng.2011.05.012.

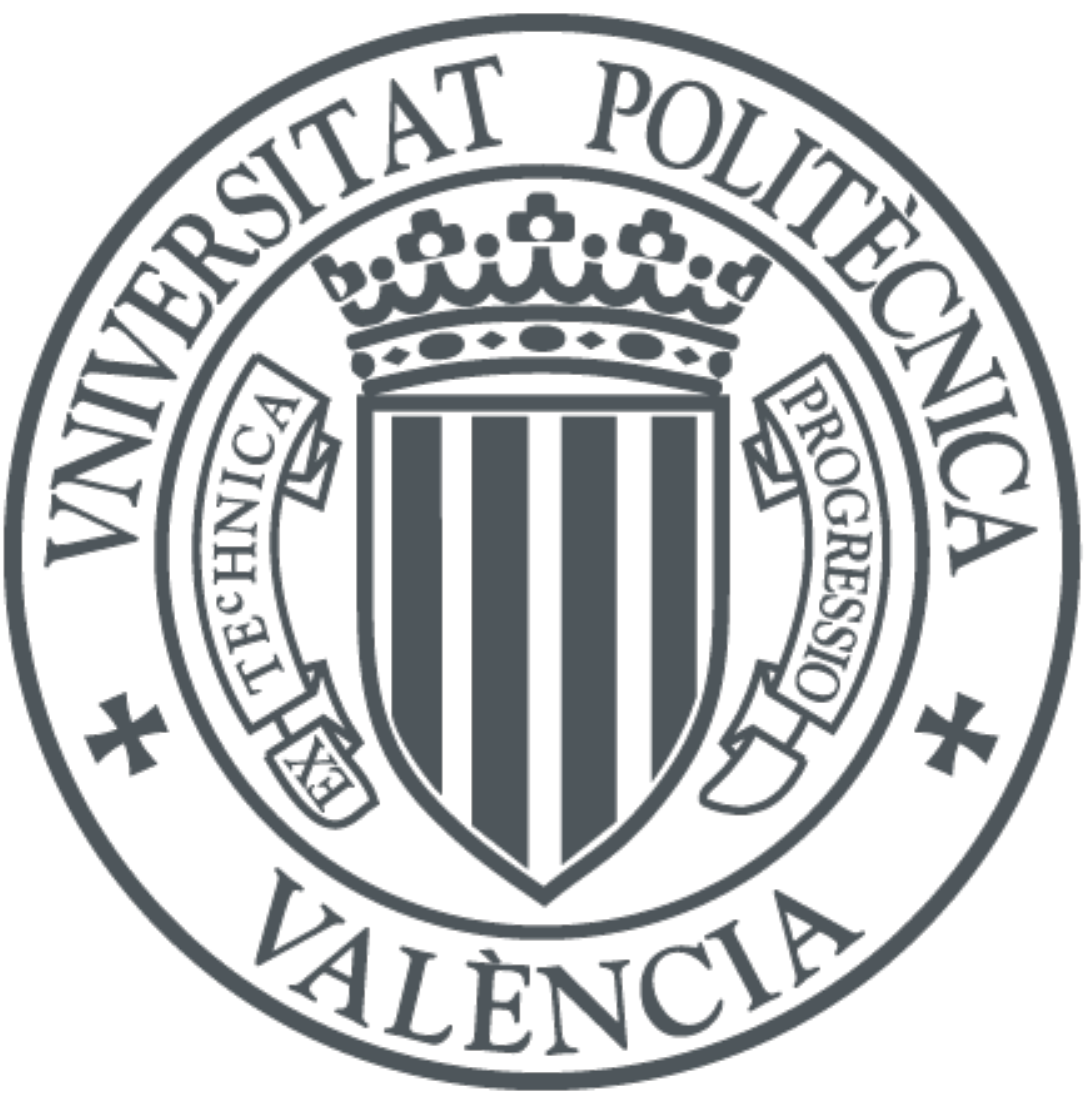

The final publication is available at

http://dx.doi.org/10.1016/j.applthermaleng.2011.05.012

Copyright Elsevier

Additional Information 


\title{
Adaptive determination of cut-off frequencies for filtering the in-cylinder pressure in Diesel engines combustion analysis
}

\author{
F. Payri, P. Olmeda*, C. Guardiola*, J. Martín* \\ CMT-Motores Térmicos, Universidad Politécnica de Valencia, Camino de Vera s/n, 46022, Valencia, \\ Spain
}

\begin{abstract}
In-cylinder pressure analysis is a key tool for engine research and diagnosis and it has been object of study from the beginning of the internal combustion engines. One of its most useful application is combustion analysis on the basis of the First Law of Thermodynamics. However, heat release law calculations use the in-cylinder pressure derivative signal. Hence, the noise is increased and pressure filtering becomes necessary to remove high frequency noise, thus allowing for accurate combustion analyses. In this work, a methodology to set the cut-off frequency of a low-pass filter is proposed. Statistical criteria are used to separate the signal from the noise through the calculation of the Discrete Fourier Transform of several consecutive in-cylinder pressures cycles. Thus, only physically meaningful information is preserved. The proposed methodology is compared with some adaptive and non-adaptive algorithms used to select the cut-off frequencies, and it shows a good ability to adapt to different engine operating conditions.
\end{abstract}

Keywords: Diesel engines, in-cylinder pressure, signal filtering, combustion analysis

\section{Introduction}

In the last years, numerous studies aiming at a deeper understanding of the physics underlying combustion in reciprocating engines have been published. The objective of such studies is twofold: to diminish pollutant emissions [1] and to increase engine performance. In this context, in-cylinder pressure measurement is considered a valuable source of information during engine development and calibration, as it provides relevant data such as peak pressure, and indicated and pumping mean effective pressures. In-cylinder pressure analysis can even be used for more complex applications, such as air mass flow estimation [2], on-line combustion detection [3], emissions control [4], noise control [5], heat tranfer [6, 7], etc.

Information is usually obtained from in-cylinder pressure signals by means of heat release analysis $[8,9]$, which provide direct information about the instantaneous combustion evolution, thus allowing for a proper characterization of the combustion process.

\footnotetext{
${ }^{*}$ Corresponding author. Tel: +34963877650 ; fax: +34963877659

Email address: pabolgon@mot.upv.es (P. Olmeda)

$U R L$ : www.cmt.upv.es (P. Olmeda )

Preprint submitted to Elsevier
}

November 3, 2015 
Among other possibilities, this characterization permits the adjustment of combustion laws, usually by means of Wiebe functions, which can be used by engine manufacturers and researchers in order to estimate the behaviour of the engine with the purpose to optimize engine design $[10,11]$. The net apparent heat release, $\mathrm{d} Q_{n}$, can be obtained from the First Law of Thermodynamics as [9]:

$$
\mathrm{d} Q_{n}=\frac{\gamma}{\gamma-1} p \frac{\mathrm{d} V}{\mathrm{~d} t}+\frac{1}{\gamma-1} V \frac{\mathrm{d} p}{\mathrm{~d} t}
$$

where $p$ is the in-cylinder pressure, $V$ is the instantaneous volume and $\gamma$ is the adiabatic coefficient.

It can be seen in (1) that the main experimental input required for heat release analysis is the measured in-cylinder pressure (and its derivative), so that the accuracy of the obtained $\mathrm{d} Q_{n}$ is strongly dependent on the quality of in-cylinder pressure acquisition and processing.

Although the measurement of in-cylinder pressure has been subject of study since the beginning of the internal combustion engine, several problems are still present:

1. Pressure referencing, both in absolute level and angular phasing [12-14].

2. Different sources of error affecting the quality of the raw pressure signal and its subsequent analysis [15].

3. Cycle-to-cycle variations, which can be observed even when the engine operates in steady conditions. This effect is due to different causes related with fuel supply, air motion, trapped mass and its composition [16, 17].

According to the previous comments, a finite number of cycles (typically between 25 and 100) are acquired and then subject to a four-step data processing consisting of: level and angle referencing, cycle averaging, and filtering. In this work, the last two issues are considered in detail.

The content of the paper is organized as follows. First, a description of different filtering techniques used in ICE applications is presented. Then, a short description of the specific test equipment used to obtain the experimental data is given in section 3 . The step-by-step methodology used to select the filter parameters is described in section 4. The validation of the proposed method in terms of repeatability is presented in section 5. Once the method is validated, it is applied to a representative set of experimental signals, the results being presented in section 6 , in which comparison with other filters is also shown. Finally, in section 7 , the most relevant conclusions extracted from the work are summarized.

\section{Filtering techniques}

Different filtering techniques may be used to obtain a smooth mean pressure cycle. Averaging diminishes point-to-point variation due to signal noise, thus becoming an easy, but usually insufficient, way to filter in-cylinder pressure. Another option is the moving average filter, but its ability to smooth depends on the sampling interval [18]; additionally, sharp pressure variations associated with premixed combustion are distorted. Slightly more complex, but more accurate, procedures are based on the use of least squares fits, such as the Savitzky-Golay filter [19]. 
However, digital filtering must usually be applied if high quality results are required. Low-pass filters are widely used as they are suitable for retaining the physical information useful for combustion analysis while removing high frequency noise. The main problem associated with low-pass filters is the determination of the optimum cut-off frequency $[20,21]$, i.e. the frequency above which the noise-to-signal ratio becomes important. Moreover, the direct elimination of the high-frequency band can cause overshoots in the pressure signal (the Gibbs effect) which cause non negligible problems in the heat release calculation. This can be mitigated by smoothing the transition with a Hanning window [18], defined between two cut-off frequencies: the stopband initial frequency, $k_{1}$ and the stopband final frequency, $k_{2}$, as shown in (2).

$$
\theta_{k}=\left\{\begin{array}{cc}
\theta_{k}=1 & k<k_{1} \\
\cos \left(\frac{\pi}{2} \cdot \frac{k-k_{1}}{k_{2}-k_{1}}\right) & k_{1} \leq k \leq k_{2} \\
\theta_{k}=0 & k>k_{2}
\end{array}\right.
$$

In most cases, the selection of the cut-off frequencies are based on ad-hoc methods and rule-based algorithms. The main objective of this paper is to propose an automatic methodology allowing to select the values of cut-off frequencies so that the signal filtering of in-cylinder pressure is optimized for combustion analysis. As the methodology is based on the own signal characteristics, no rule-based criterion is necessary. The performance of the filter will be compared with other filters in order to illustrate the improvement achievable in terms of heat release. Although the filtering procedure will be applied to experimental data from direct injection diesel engines, the proposed approach is easily adaptable to other engines and measurement configurations.

\section{Experimental set-up and tests}

A sketch of the test cell layout with the basic instrumentation is shown in Figure 1. Measurements were carried out on a high-speed direct-injection 2.0 litre diesel engine currently in production, whose main characteristics are given in Table 1. The engine was directly coupled to an electric dynamometer allowing for engine speed an load control.

Mean variables necessary for controlling the engine operating point and also for combustion analysis were acquired at a constant sample rate of $100 \mathrm{~Hz}$ with an AVL test system. In-cylinder pressure was measured in one of the four cylinders by means of a Kistler $6055 \mathrm{~B}$ glow-plug piezoelectric transducer connected to a Kistler 5015 charge amplifier. Measurements were performed with an angular resolution of 0.5 crankangle degrees and a Yokogawa DL708E oscillographic recorder was used. The pressure sensor was calibrated according to the usual method [22] based on a quasi-steady calibration by means of a deadweight tester with NPL and NIST traceability. With this procedure, the relative error obtained from the calibration of the transducer was $\pm 0.7 \%$.

Table 2 summarizes the tests used for illustrating the results of the proposed methodology, which include a wide variety of engine operating conditions: motored tests at different engine speeds and also several combustion tests with different engine speeds and loads. 


\section{Methodology for the determination of the cut-off frequencies}

The method used to determine the cut-off frequencies is based on the qualification of the frequencies according to their noise-to-signal ratio. The procedure is as follows:

1. The starting point is the raw in-cylinder pressure signal measured during $n_{c}$ consecutive cycles at $n_{s}$ samples per cycle. Neither the reference level nor the sensor sensitivity are required at this point, since the first only affects the signal mean value while the second is just a linear conversion.

2. The Discrete Fourier Transform, DFT, is applied to all the consecutive cycles, thus obtaining $n_{c} \cdot n_{s}$ complex numbers, $A_{i}$, given by:

$$
A_{i}=A_{i}^{\mathrm{Re}}+\mathrm{j} A_{i}^{\mathrm{Im}} \quad \text { with } \quad i=1,2 \ldots n_{c} \cdot n_{s}
$$

corresponding to the frequencies, $f_{i}$, given by:

$$
f_{i}=\frac{i-1}{n_{c} \cdot n_{s}} f_{s}=\frac{i-1}{n_{c}} f_{0} \quad \text { with } \quad i=1,2 \ldots n_{c} \cdot n_{s}
$$

where $f_{s}$ is the sampling frequency and $f_{0}$ is the frequency of the engine thermodynamic cycle. For convenience, the frequency $f_{i}$ is normalized by dividing it by $f_{0}$ :

$$
f_{i}^{n}=\frac{f_{i}}{f_{0}}=\frac{i-1}{n_{c}} \quad \text { with } \quad i=1,2 \ldots n_{c} \cdot n_{s}
$$

where $f_{i}^{n}$ is the normalized frequency of the $i$-th harmonic.

3. Two kind of harmonics can be now considered in the spectrum:

(a) On one hand, those harmonics for which $f_{i}^{n}$ is a natural number. These harmonics will be called basic harmonics because they contain the main information on the average cycle, which will be denoted as:

$$
S_{k}=S_{k}^{\mathrm{Re}}+\mathrm{j} S_{k}^{\mathrm{Im}} \quad \text { with } \quad k=1, n_{c}+1,2 n_{c}+1 \ldots\left(n_{s}-1\right) n_{c}+1
$$

As it can be seen in the top part of Figure 2, the amplitude of $S_{k}$ is higher than that of the rest of harmonics at low and middle frequencies (normalized frequencies lower than 50), where the main contribution of compressionexpansion and combustion to the signal is found.

(b) On the other hand, in the bottom part of Figure 2, it can easily be seen that between two consecutive basic harmonic there are $n_{c}-1$ non-basic harmonics with a lower gain (at least at low an middle frequencies) which may be regarded as noise:

$$
N_{k, m}=N_{k, m}^{\mathrm{Re}}+\mathrm{j} N_{k, m}^{\mathrm{Im}}=A_{(k-1) \cdot n_{c}+m+1} \quad \text { with } \quad\left\{\begin{array}{l}
k=1,2 \ldots n_{s} \\
m=1,2 \ldots n_{c}-1
\end{array}\right.
$$

The next step is to assign to each basic frequency the noise associated with its neighbouring frequencies. This is accomplished by assuming that all the non-basic harmonics between two consecutive basic harmonics represent the noise related to the first basic harmonic of the range, as indicated in the bottom part of Figure 2. At this point one has $n_{s}$ sets of harmonics consisting of one basic harmonic and its $n_{c}-1$ associated noise harmonics: 


$$
\left\{S_{k},\left\{N_{k, m}\right\}\right\} \quad \text { with } \quad\left\{\begin{array}{l}
k=1,2 \ldots n_{s} \\
m=1,2 \ldots n_{c}-1
\end{array}\right.
$$

For each $k$, the mean value of the $n_{c}-1$ harmonics $\left\{N_{k, m}\right\}$ will be denoted as $N_{k}$; where $N_{k}=N_{k}^{\mathrm{Re}}+\mathrm{j} N_{k}^{\mathrm{Im}}$.

4. The statistical method applied to determine the values of the cut-off frequencies is as follows: for each $k$, it is assumed that both the real and imaginary parts of $S_{k}$ and its associated noise $\left\{N_{k, m}\right\}$ are independent variables whose populations follow a Gaussian distribution. Accordingly, for the real part of the harmonics one may write:

$$
\left.\begin{array}{l}
S_{k}^{\mathrm{Re}} \equiv N\left(\hat{S}_{k}^{\mathrm{Re}}, \sigma_{k}^{\mathrm{Re}}\right) \\
N_{k}^{\mathrm{Re}} \equiv N\left(0, \sigma_{k}^{\mathrm{Re}} / \sqrt{n_{c}-1}\right)
\end{array}\right\} \quad \text { with } \quad k=1,2 \ldots n_{s}
$$

where $\hat{S}_{k}^{\mathrm{Re}}$ is the real mean value of the real part of the basic harmonic at frequency $f_{k}$, and $\sigma_{k}^{\mathrm{Re}}$ is the standard deviation of the real part of the noise harmonics, which is assumed to be equal [23] for $S_{k}$ and $N_{k}$, because they are affected by the same error sources. It is important to highlight that the real values $\hat{S}_{k}^{\mathrm{Re}}$ and $\sigma_{k}^{\mathrm{Re}}$ are unknown and only their respective estimates $S_{k}^{\mathrm{Re}}$ and $s_{k}^{\mathrm{Re}}$ are available. Notice in (9) that the mean value of the real part of the noise harmonics is zero, as stated above.

At this point the key issue is to determine whether or not $\hat{S}_{k}^{\mathrm{Re}}$ is statistically different from the noise $N_{k, m}$. Following the steps detailed in the appendix, a simple statistical analysis was performed to ensure that the mean value of the real part of the basic harmonics $\hat{S}_{k}^{\mathrm{Re}}$ and the mean value of the imaginary part of the basic harmonics, $\hat{S}_{k}^{\mathrm{Im}}$, lie within the confidence intervals:

$$
\begin{aligned}
& \left(S_{k}^{\mathrm{Re}}-N_{k}^{\mathrm{Re}}\right) \pm s_{k}^{\mathrm{Re}} \cdot t_{n_{c}-2}^{\alpha / 2} \sqrt{n_{c} /\left(n_{c}-1\right)} \\
& \left(S_{k}^{\operatorname{Im}}-N_{k}^{\operatorname{Im}}\right) \pm s_{k}^{\operatorname{Im}} \cdot t_{n_{c}-2}^{\alpha / 2} \sqrt{n_{c} /\left(n_{c}-1\right)}
\end{aligned}
$$

with a probability $(1-\alpha)$; here, the symbol $t$ represents a Student's distribution, $s_{k}^{\operatorname{Im}}$ denotes the sample variance of the imaginary part of $\left\{N_{k, m}\right\}$. The two confidence intervals defined by (10) determine a rectangular area in the complex plane, in which the real mean value of the $k$-th basic harmonic is comprised with a probability $(1-\alpha)$.

The cut-off frequencies $k_{1}$ and $k_{2}$ are chosen with the criterion that the confidence rectangles in the complex plane obtained with a probability of $98 \%$ and $80 \%$, respectively, contain the origin. These conditions are equivalent to state that there is no statistical difference between the signal and the noise at a given frequency. Figure 3 shows an example of the determination of $k_{1}$. The complete spectrum is plotted in the top part of Figure 3 while in the bottom part two confidence rectangles, one corresponding to a harmonic, $S_{43}$, which does not fulfil the conditions (10) and the other to first harmonic for which the conditions are accomplished, are plotted. In this case, the normalized cut-off frequency is $k_{1}=44$.

At this point, two processings are applied to the signal spectrum in order to obtain a filtered mean cycle: on one hand, signal averaging is performed by selecting only the basic harmonics, and on the other hand high frequency signal noise is eliminated by applying the described low-pass filter. 


\section{Method repeatability}

The first issue explored was the repeatability of the methodology with a double purpose: a reasonable repeatability must be ensured in view of the final application of the methodology, and repeatability can be used as a criterion for determining the minimum size of the sample to be considered.

Sets of 25 consecutive cycles were acquired for given engine operating conditions. Then the set was divided into different subsets, with sizes ranging from 5 consecutive cycles to 25 , and shifting the initial cycle considered. Hence, for each subset size different subsets were available, and error metrics could be determined.

Figure 4 shows the mean value and the standard deviation of $k_{1}$ and $k_{2}$ obtained when changing the number of consecutive cycles considered. Frequency $k_{1}$ exhibits a clear trend to stabilization: for 13 or more cycles, its mean value reaches a value of 70 , and the standard deviation also reaches a quite constant value below \pm 1 . Such a statistical analysis allows, for fixed operation conditions, to determine the number of cycles to be considered: in this case 13 cycles. Nevertheless, since this characteristic depends on the engine and the operating conditions, it is not possible to derive an absolute rule, but only a procedure.

Concerning $k_{2}$, a more erratic behaviour is observed. In this case, the standard deviation does not decrease when the number of cycles is increased. However, the mean value is quite constant for all repetitions, and also this cut-off frequency has a moderate influence within its variation interval, as it is demonstrated in Figure 5. Hence, it was decided to choose the number of cycles uniquely on the basis of the behaviour of $k_{1}$, thus neglecting the influence of the sample size on $k_{2}$.

Finally, the proposed methodology was compared with the direct averaging of the signal, as shown in Figure 6. It is interesting to note that the pressure signal spectra clearly depend on the engine load, but not monotonically: the advisable cut-off frequency for the $25 \%$ load condition almost triplicates the value of that obtained at full load operation (212 vs. 72 harmonics), while pressure in motored conditions shows a lower value (51 harmonics). This important variation is due to the characteristics of the combustion development. In the low frequency region (approximately the first 25 basic harmonics) the three signals show a similar behaviour, because this part of the spectra is essentially dominated by the compression-expansion effects. The middle frequency region is the most important part regarding combustion, whereas the filter completely eliminates the high frequency contents attributed to noise. Slight differences between averaged and filtered spectra can be seen in the filter transition band (normalized frequencies between 70 and 80 at full load) due to the filter characteristics. When comparing the reconstructed incylinder pressure no significant differences could be appreciated in all the cases; however, the high frequency content strongly affect, except in motoring conditions, the in-cylinder pressure derivative, which is used for calculating the heat release law, and it is verified that the proposed methodology is able to smooth the signal with an automated selection of the filtering cut-off frequencies.

\section{Filter application}

Once the filtering methodology was validated in terms of repeatability, its performance has been compared with other available filters in different engine operating con- 
ditions (see Table 2). The advantage of the adaptive method is expected to be clear in combustion tests (see section 5), where the rates of heat release obtained with an in-house thermodynamic model [24] will be used.

First, Figure 7 shows the spectra (left) obtained from the averaged signal and three different Savistzky-Golay filters [19], using a cubic polynomial fitting where the numbers in the legend indicate the number of points, $n_{p}$, used for signal fitting. Two important conclusions can be drawn:

- The higher is $n_{p}$, the lower is the high frequency noise of the rate of heat release. However, as $n_{p}$ increases the Savistzky-Golay filter also affects the mid and low frequencies which contain significant information on combustion. It is thus very difficult to remove efficiently the noise while retaining the combustion information. For the test shown in Figure 7 , the noise is well removed with $n_{p}=27$, but the combustion peak is also smoothed. This behavior is critical in case of sharp heat release peaks such as those found in premixed combustions. Therefore, smoothing methods that are not frequency sensitive are not recommendable.

- Even in case that a suitable balance could be reached between noise removal and physical information conservation, the optimal filter parameter $n_{p}$ could be very different at other operating points, so that a general rule to set it cannot be formulated.

Two digital filters were selected to perform the comparison with the proposed approach:

1. A mapped cut-off frequency filter proposed by Martín [25], which used a criterion based only on engine speed, varying $k_{1}$ linearly from 210 at $1250 \mathrm{rpm}$ to 114 at 4000 $\mathrm{rpm}$, and setting $k_{2}=k_{1}+60$. However, the frequency band of the pressure signal can vary significantly at different operating conditions and not only on engine speed. As an example, in Figure 8 the $k_{1}$ values obtained with the proposed methodology in tests performed at different engine speeds and loads are shown, and significant variations can be observed.

2. An adaptive filter proposed by Kosarev [23], in which it is assumed that the right half of the spectrum is noise; the cut-off frequency is set so that the ratio between the harmonic level and the standard deviation of the noise is unity.

Figure 9 shows the rate of heat release obtained with the three methods at 1750 rpm. At low load, the proposed filter behaves similarly to the Kosarev filter, despite the important difference in their cut-off frequencies. This difference is due to the criterion used to determine when the signal and the noise have similar gains. The proposed method seems to underestimate $k_{1}$ since normalized frequencies between 175 and 260, in which the basic harmonics show an increment, are removed. However, the effect is almost negligible, a possible explanation being that in both cases $k_{1}$ coincides with a minimum of the spectrum and thus the Gibbs effect is attenuated. This justification is confirmed by the results of the fixed cut-off frequency filter, in which an intermediate $k_{1}$ is used with worse performance than for the two adaptive filters, showing a higher oscillation level during the pilot combustion. Finally, at high load the proposed filter is able to eliminate the oscillations associated with the excessive value of $k_{1}$ provided by the other two filters. 
Figure 10 shows the rate of heat release obtained from an engine speed sweep at full load and a load sweep at $1750 \mathrm{rpm}$. As it can be seen, even though quite different combustions take place the proposed methodology is able to adapt properly the filter parameters to all the operating conditions. In particular, very small oscillations remain in the combustion laws. Regarding the other filters, it has been checked (not plotted) that their performance is similar to that indicated by the results previously shown: the Savistzky-Golay filter does not behave suitably in most of the cases, the mapped cut-off frequency filter behaves in a reasonable way only for some operating conditions, and the performance of the adaptive Kosarev filter is similar, but not better in any case, than that of the proposed flter.

\section{Conclusions}

A methodology for automatically determining cut-off frequencies for the filtering of the in-cylinder pressure signal of internal combustion engines has been presented. The methodology is based on the statistical analysis of the DFT representation of the signal: signal-to-noise ratio is identified and used for detecting the frequency where the contribution of the noise equals that of the signal.

The proposed method exhibits a high repeatability and it is able to adapt suitably the cut-off frequencies to the bandwidth of the pressure signal. Hence, the methodology can be used for the full operating engine range, without the need of additional manual settings. The proposed filter has been compared with other filters, both adaptive and non-adaptive. It has been demonstrated that the ability of the filter to set automatically the cut-off frequencies is an important issue, and that the performance of the proposed approach is clearly better than that of the classical fixed filters and rule-based filters. The results obtained have been shown to be similar to those given by another adaptive in most operating points; however, the criterion used in the proposed filter appears to be more robust in some cases, and oscillations in the rate of heat release are better removed. This allows for a more precise analysis of the combustion process and for obtaining more accurate combustion laws.

In addition, it has been shown that the statistical analysis of the cut-off frequency for different numbers of measured cycles can be used for the automatic selection of the number or cycles to be measured, thus optimizing the size of the sample.

\section{Acknowledgements}

The authors thank the Universidad Politécnica de Valencia (PAID-06-09) and Generalitat Valenciana $(\mathrm{GV} / 2010 / 045)$ for its valuable support to this work.

[1] A. Broatch, J.M. Luján, S. Ruiz, P. Olmeda, Measurement of hydrocarbon and carbon monoxide emissions during the starting of automotive DI Diesel engines, Int. J. Automot. Technol. 9 (2008) 129-140.

[2] J.M. Desantes, J. Galindo, C. Guardiola, V. Dolz, Air mass flow estimation in turbocharged diesel engines from in-cylinder pressure measurement, Exp. Therm. Fluid Sci. 34 (2010) 37-47.

[3] J.M. Luján, V. Bermúdez, C. Guardiola, A. Abbad, A methodology for combustion detection in diesel engine through in-cylinder pressure derivative signal, Mech. Syst. Signal Pr. 24 (2010), 2261-2275.

[4] M. Beasley, R. Cornwell, P. Fussey, R. King, A. Noble, T. Salamon, A. Truscott, Reducing diesel emissions dispersion by coordinated combustion feedback control, SAE paper 2006-01-0186 (2006). doi: $10.4271 / 2006-01-0186$ 
[5] F. Payri, A. Broatch, B. Tormos, V. Marant, New methodology for in-cylinder pressure analysis in direct injection diesel engines - application to combustion noise, Meas. Sci. Technol. 16 (2005) $540-547$.

[6] A.J. Torregrosa, P. Olmeda, J. Martín, C. Romero A Tool for Predicting the Thermal Performance of a Diesel Engine. Heat Transf. Eng., doi: 10.1080/01457632.2011.548639 (2011).

[7] J.M. Desantes, A.J. Torregrosa, A. Broatch, P. Olmeda, Experiments on the influence of intake conditions on local instantaneous heat flux in reciprocating internal combustion engines, Energy 36 (2011), 60-69.

[8] M. Lapuerta, O. Armas, J.J. Hernández, Diagnosis of DI diesel combustion from in-cylinder pressure signal by estimation of mean thermodynamic properties of the gas, Appl. Therm. Eng. 19 (1999) 513-529.

[9] J.B. Heywood, Internal Combustion Engine Fundamentals, McGraw-Hill, New York, 1988.

[10] M. Torres Garcia, F.J. Jiménez-Espadafor Aguilar, J.A. Becerra Villanueva, E. Carvajal Trujillo, Analysis of a new analytical law of heat release rate (HRR) for homogenous charge compression ignition (HCCI) combustion mode versus analytical parameters. Appl. Therm. Eng. 31 (2011) $458-466$

[11] J. Galindo, H. Climent, B. Plá, V.D. Jiménez. Correlations for wiebe function parameters for combustion simulation in two-stroke small engines, Appl. Therm. Eng. 31 (2011), 1190-1199.

[12] K. Lee, M. Yoon, M. Sunwoo, A study on pegging methods for noisy cylinder pressure signal, Con. Eng. Prac. 16 (2008) 922-929.

[13] H. Chang, Y. Zhang, L. Chen, An applied thermodynamic method for correction of TDC in the indicator diagram and its experimental confirmation, Appl. Therm. Eng. 25 (2005) 759-768.

[14] E. Pipitone, A. Beccari, Determination of TDC in internal combustion engines by a newly developed thermodynamic approach, Appl. Therm. Eng. 30 (2010) 1914-1926.

[15] D.T. Hountalas, A. Anestis, Effect of pressure transducer position on measured cylinder pressure diagram of high speed diesel engines, Energy Conv. Manag. 39 (1998) 589-607.

[16] F. Payri, J.M. Luján, C. Guardiola, G. Rizzoni, Injection diagnosis through common-rail pressure measurement, Proc. Inst. Mech. Eng. Part D-J. Automob. Eng. 220 (2006) 347-357.

[17] J.M. Luján, J. Galindo, J.R. Serrano, B. Pla, A methodology to identify the intake charge cylinderto-cylinder distribution in turbocharged direct injection diesel engines, Meas. Sci. Technol. 19, 065401 (2008).

[18] S.X. Shi, H.Z. Sheng, Numerical simulation and digital signal processing in measurements of cylinder pressure of internal combustion engines, Proc. Inst. Mech. Eng. International Conference on Computers in Engine Technology (C345) C20/87 (1987) 211-218.

[19] A. Savitzky, M.J.E. Golay, Smoothing and differentiation of data by simplified least squares procedures, Anal. Chem. 36 (1964) 1627-1639.

[20] F. Payri, F.M. Luján, J. Martín, A. Abbad, Digital signal processing of in-cylinder pressure for combustion diagnosis of internal combustion engines, Mech. Syst. Signal Pr. 24 (2010), 1767-1764.

[21] G. Giakas, V. Baltzopoulos, Optimal digital filtering requires a different cut-off frequency strategy for the determination of higher derivatives, J. Biomech. 30 (1997) 851-855.

[22] J. Tichy, G. Gautschi, Piezoelektrische Messtechnik, Springer Verlag, Berlin, 1980.

[23] E.L. Kosarev, E. Pantos, Optimal smoothing of 'noisy' data by fast Fourier transform, J. Phys. E: Sci. Instrum. 16 (1983) 537-543

[24] F. Payri, S. Molina, J. Martín, O. Armas, Influence of measurement errors and estimated parameters on combustion diagnosis, Appl. Therm. Eng. 26 (2006) 226-236.

[25] J. Martín, Aportación al diagnóstico de la combustión en motores Diesel de inyección directa, Editorial Universidad Politécnica de Valencia, Valencia (Spain), 2007. 
Figures

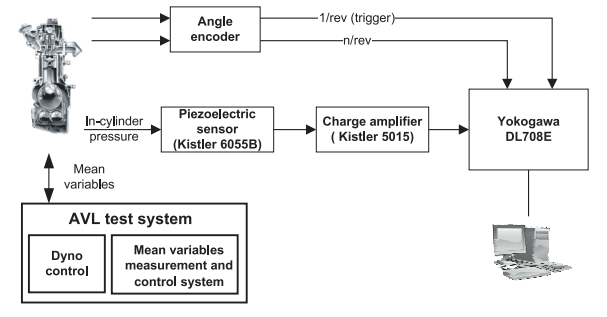

Figure 1: Measurement chain layout in the engine test bench. 


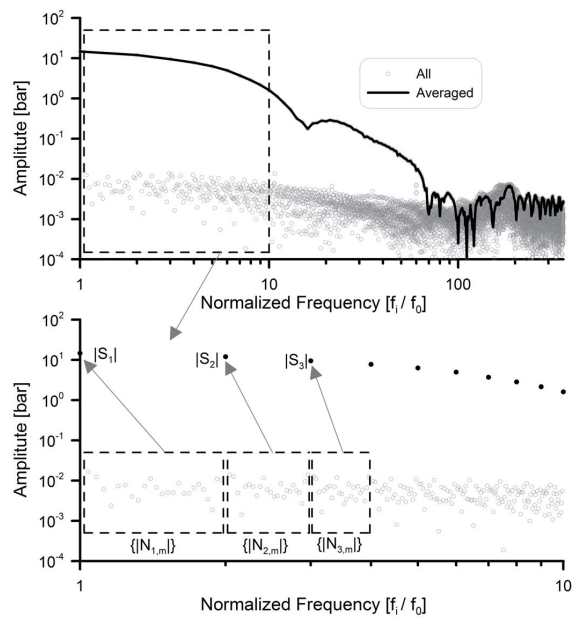

Figure 2: Complete Fourier spectrum of 25 cycles (top) and noise attribution scheme for the DFT components of the signal (bottom). 


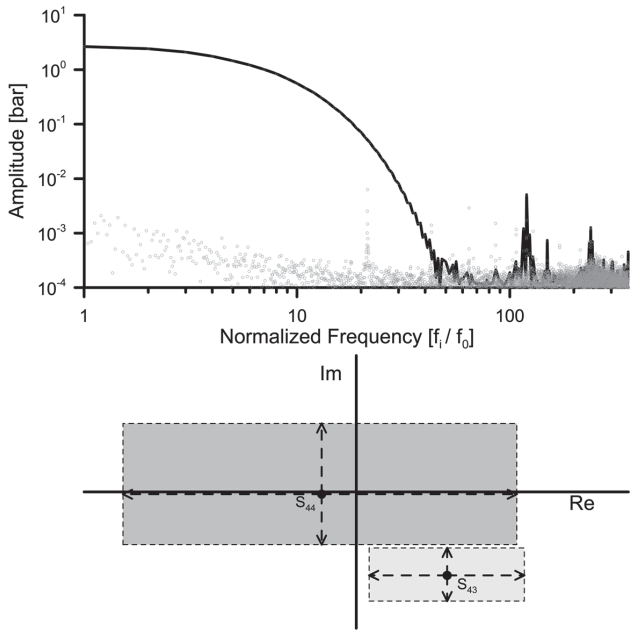

Figure 3: Fourier spectrum of the $1000 \mathrm{rpm}$ and motored (top) and an example of the determination of $k_{1}$ using equations (11) and (12). 

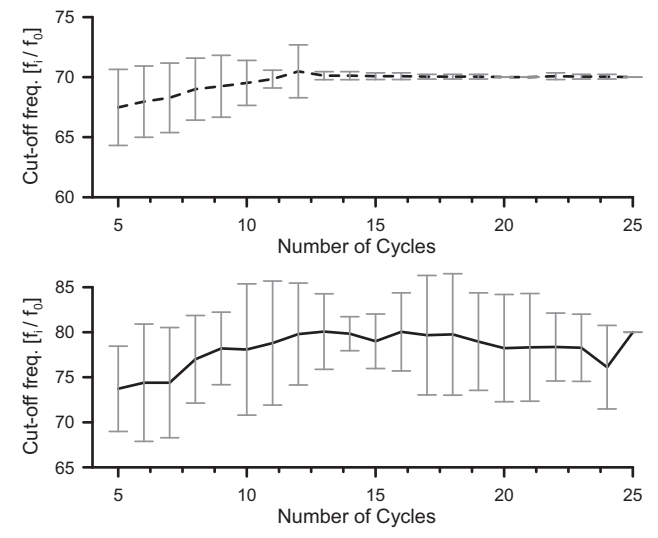

Figure 4: Mean value of the cut-off frequencies (black) and their standard deviation (grey) attending the number of cycles considered, where the top part corresponds to initial frequency $k_{1}$ and bottom part to the final frequency $k_{2}$. Data corresponds to test of $2000 \mathrm{rpm}$ and full load. 

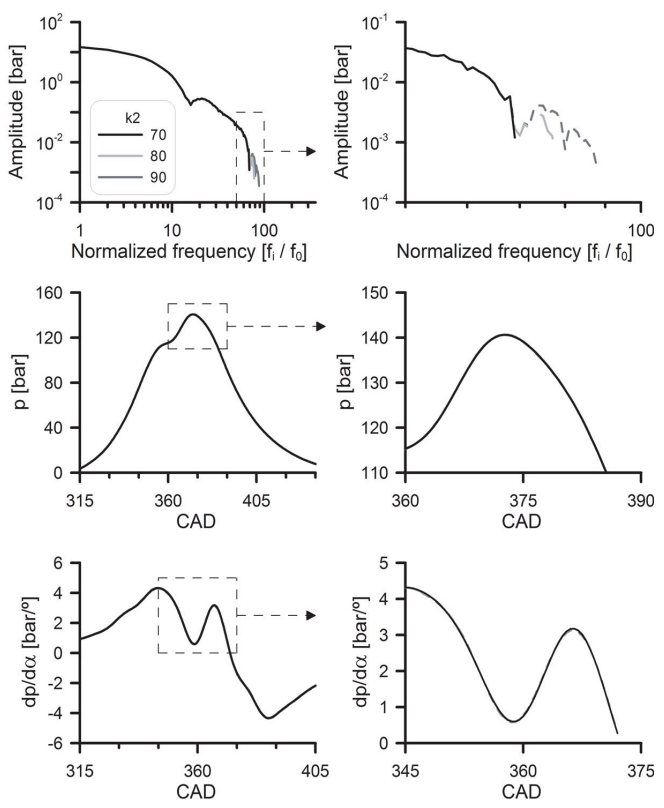

Figure 5: Effect of varying $k_{2}$ for a fixed value of $k_{1}$ on Fourier spectrum (top), pressure (medium) and pressure derivative (bottom). 


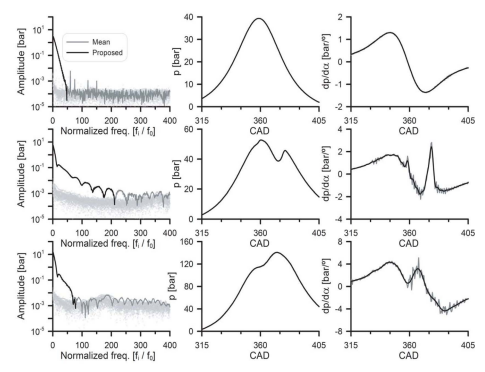

Figure 6: Filter application at $2000 \mathrm{rpm}$ and three different loads: motored (top), $25 \%$ load (centre) and full load (bottom). $k_{1}$ cutt-off frequencies are: 51, 212 and 72 respectively. 

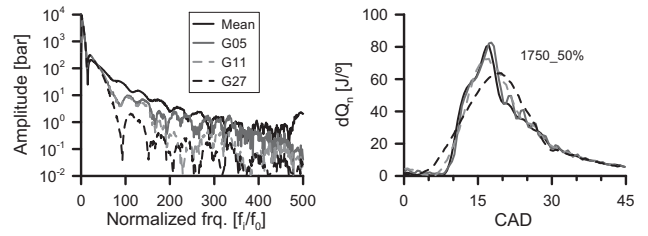

Figure 7: Fourier spectra (left) and rate of heat release (right) at $1750 \mathrm{rpm}$ and $50 \%$ load obtained with the Savitzky-Golay filter using different number of points. 


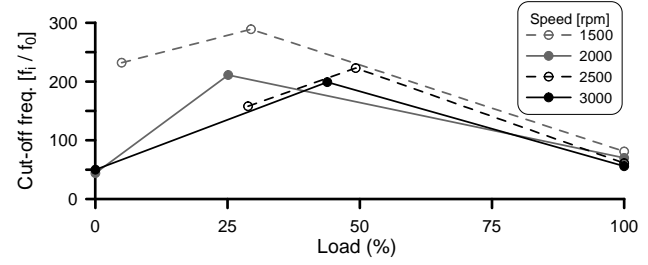

Figure 8: Cut-off frequencies for different engine speeds and loads. 

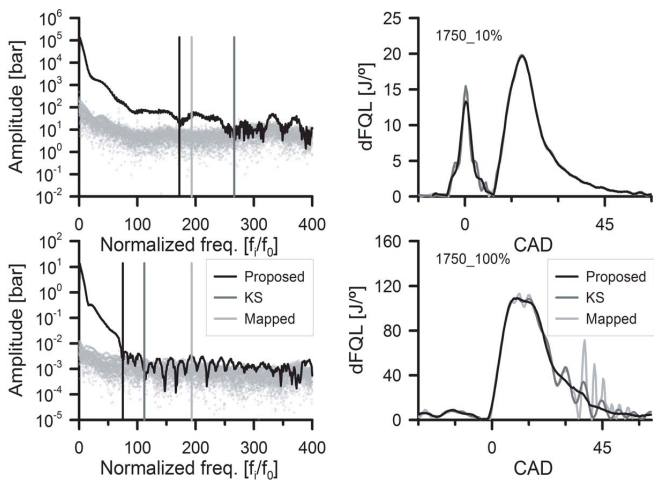

Figure 9: Fourier spectra (left) and rate of heat release (right) at $1750 \mathrm{rpm}$ and $10 \%$ load (top) and full load (bottom) obtained with a mapped, Kosarev filter (KS) and the proposed filter. 

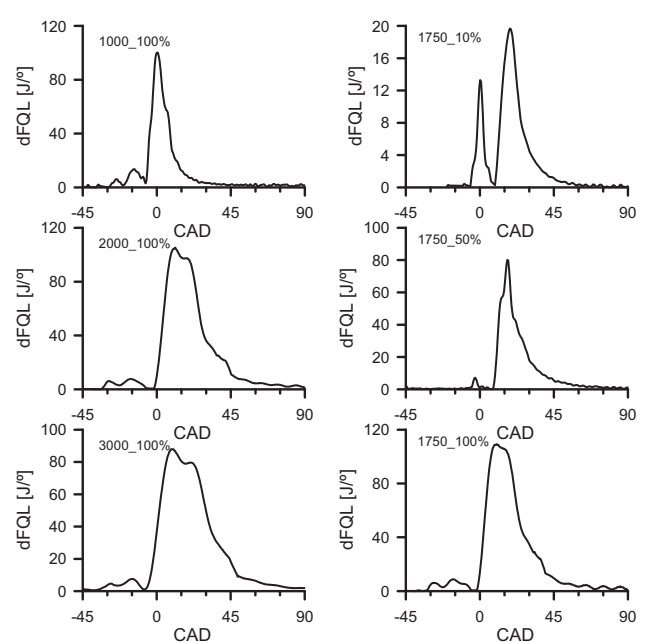

Figure 10: Rate of heat release at different operating points applying the proposed methodology. 
Tables

\begin{tabular}{lc}
\hline Displaced volume & $2.0 \mathrm{l}$ \\
Cylinders & 4 \\
Bore & $84 \mathrm{~mm}$ \\
Stroke & $90 \mathrm{~mm}$ \\
Connecting rod length & $143.5 \mathrm{~mm}$ \\
Compression ratio & $16: 1$ \\
Maximum power & $125 \mathrm{~kW}$ \\
\hline
\end{tabular}

Table 1: Main engine characteristics

\begin{tabular}{|c|c|}
\hline $\begin{array}{l}\text { Speed } \\
{[\text { rpm }]}\end{array}$ & $\begin{array}{c}\text { Load } \\
{[\%]}\end{array}$ \\
\hline $1000-5000$ & 0 (motoring) \\
\hline 1000 & 100 \\
\hline 1500 & $5,30,100$ \\
\hline 1750 & $10,50,100$ \\
\hline 2000 & 25,100 \\
\hline 2500 & $30,50,100$ \\
\hline 3000 & 44,100 \\
\hline
\end{tabular}

Table 2: Experimental tests 


\section{Annex: description of the statistical methodology}

Consider a set of harmonics composed of one basic harmonic and its $n_{c}-1$ associated noise harmonics:

$$
\left\{S_{k},\left\{N_{k, m}\right\}\right\}_{k} \quad \text { with }\left\{\begin{array}{l}
k=1,2 \ldots n_{s} \\
m=1,2 \ldots n_{c}-1
\end{array}\right.
$$

To determine whether or not the basic harmonic is statistically different from the noise harmonics the following process is followed.

As justified both the real and imaginary parts of $S_{k}$ (signal) and its associated noise $\left\{N_{k, m}\right\}$ are independent variables which populations follow a Gaussian distribution. For the real part of the harmonics the expression (9) was proposed. As the difference of two Gaussian distribution is also a Gaussian distribution:

$$
S_{k}^{\operatorname{Re}}-N_{k}^{\operatorname{Re}} \equiv N\left(\hat{S}_{k}^{\operatorname{Re}}, \sigma_{k}^{\operatorname{Re}} \sqrt{n_{c} /\left(n_{c}-1\right)}\right) \quad \text { with } \quad k=1,2 \ldots n_{s}
$$

Standardising it is obtained:

$$
\left(S_{k}^{\mathrm{Re}}-N_{k}^{\mathrm{Re}}-\hat{S}_{k}^{\mathrm{Re}}\right) / \sigma_{k}^{\mathrm{Re}} \sqrt{n_{c} /\left(n_{c}-1\right)} \equiv N(0,1) \text { with } k=1,2 \ldots n_{s}
$$

and thus, $\hat{S}_{k}^{\mathrm{Re}}$ is in the confidence interval

$$
\left(S_{k}^{\mathrm{Re}}-N_{k}^{\mathrm{Re}}\right) \pm Z_{\alpha / 2} \cdot \sigma_{k}^{\mathrm{Re}} \sqrt{n_{c} /\left(n_{c}-1\right)}
$$

with a probability $(1-\alpha)$, being $Z_{\alpha / 2}$ the probability of a Gaussian distribution.

Taking into account that the ratio between the sample variance $s_{k}^{2}$ and the population variance $\sigma_{k}^{2}$ of a Gaussian distribution follow a $\chi^{2}$ distribution, the following expressions can be written:

$$
\left(n_{c}-2\right)\left(s_{k}^{\mathrm{Re}} / \sigma_{k}^{\mathrm{Re}}\right)^{2} \equiv \chi_{n_{c}-2}^{2}
$$

where $s_{k}^{\mathrm{Re}}$ is the sample variance of the real part of $\left\{N_{k, m}\right\}$.

Considering the definition of the Student's $t$ distribution, the following expression can be found from (13) and (15):

$$
\frac{\left(S_{k}^{\mathrm{Re}}-N_{k}^{\mathrm{Re}}-m_{s}^{\mathrm{Re}}\right) / \sigma_{k}^{\mathrm{Re}} \sqrt{n_{c} /\left(n_{c}-1\right)}}{\sqrt{\left(n_{c}-2\right)\left(s_{k}^{\mathrm{Re}} / \sigma_{k}^{\mathrm{Re}}\right)^{2} /\left(n_{c}-2\right)}} \equiv t_{n_{c}-2}
$$

thus, the mean value of real part of the basic harmonics population, $\hat{S}_{k}^{\mathrm{Re}}$, is in the confidence interval

$$
\left(S_{k}^{\mathrm{Re}}-N_{k}^{\mathrm{Re}}\right) \pm s_{k}^{\mathrm{Re}} \cdot t_{n_{c}-2}^{\alpha / 2} \sqrt{n_{c} /\left(n_{c}-1\right)}
$$

with a probability $(1-\alpha)$. 